\title{
Hierarchical Wireless Network Architecture for Distributed Applications
}

\author{
Zeashan Hameed Khan \\ Control Systems Department \\ GIPSA-lab, Grenoble, France \\ zeashan-hameed.khan@ @ipsa-lab.inpg.fr
}

\author{
Denis Genon Catalot \\ Networks \& Telecom Department \\ LCIS, Valence, France \\ Denis.Genon-Catalot@iut-valence.fr
}

\author{
Jean Marc Thiriet \\ Control Systems Department \\ GIPSA-lab, Grenoble, France \\ jean-marc.thiriet@gipsa-lab.inpg.fr
}

\begin{abstract}
This paper describes a hierarchical wireless network architecture for real time remote coordination and monitoring in distributed applications. The problem of defining a communication architecture keeping in view the constraints imposed by control and monitoring tasks requires a co-design approach. The present framework takes into account the available infrastructure to satisfy the requirements of end to end delay and quality of service for time critical applications communicating over network.
\end{abstract}

Keyword; Network architecture, Distributed monitoring, QoS adaptation, Realtime coordination

\section{INTRODUCTION}

Wireless communication networks are the preferred technology compared to wired networks as it offers more flexibility and lower cost for installation and commissioning. Wireless links are commonly used for long-range communication in SCADA systems, however, in order to extend wireless networks for remote control, coordination and applications involving emergency alerts, strict requirements on availability, robustness, reliability and performance have to be satisfied in order to meet application requirements and industrial standards [1]. In this paper, we consider the utilization of heterogeneous wireless networks for communication needs of long range monitoring by generating event based alarms that can be extended to periodic synchronization signaling between autonomous systems located far away. The advantages of distributed sensing, control and actuation are constrained by the communication variable, which is further dependent on the distance between the mobile clusters and the control station, channel bandwidth available, cost of infrastructure, quality of service (QoS), etc. [2]. For wireless networks, the communication challenges are two-fold due to interference, fading and multi path effects which are inherent to wireless technology. Thus it is important to know the limitations of the wireless network architecture and the requirements imposed by the application using it so that a co-design approach can be followed [3]. Some open questions in the perspective of co-design problem include e.g., how to systematically derive or mathematically approximate the mapping between the quality of control (QoC) in distributed application and the QoS of a heterogeneous network [4, 5]. A further question evolves about how to design an adaptable QoS profile that is available at both the transmitting as well as receiving end to support a networked control system (NCS) with possible compensation in case of deterioration in network QoS.

It is important to intelligently design the communication architecture for the flow of information between the entities connected to a network (whether sensors, actuators or process itself). One approach to reduce the time delays for rapid supervision is to dedicate a part of distributed intelligence in the proximity of the application e.g., by utilizing wireless sensor networks with local data logging and processing for alarms and monitoring. However, communication needs in distributed applications are geographically distributed making network architecture a key performance parameter.

Next Generation Network (NGN) is aimed to provide packet-based telecommunication services by making use of multiple broadband, QoS-enabled transport technologies in which QoS functions are independent from underlying transport-related technologies [5]. The challenges posed by integrating different protocols in NGN and the mapping between application QoS performance specification and network QoS available in different protocols is thus simplified with emphasis on IPv6 and MPLS as key end-to-end QoS parameters without taking care of multiple transport technologies [6, 7]. For control engineers, choosing an optimal QoS profile to support a distributed networked application is a challenge in case the information passes through heterogeneous networks [7]. We will not be discussing the requirements and architectures (which include the mechanisms and interfaces) for supporting end-to-end QoS in the NGN; instead an evaluation based approach is used to characterize long distance wireless network architecture.

This paper is organized as follows: Section II presents the related work. A multi-tier generalized architecture is discussed in Section III followed by the QoS of the proposed scheme in Section IV. In Section V, application scenarios are discussed with the simulation of 2 tier architecture in section VI. In the last Section, future work directions are outlined.

\section{RELATED WORK}

Wireless network architecture is an important consideration for time critical distributed applications [8]. A comparison of flat and hierarchical architecture with adhoc wireless sensor network at the bottom is presented in [9]. The flat architecture being the highly connected network, has the scalability issue, i.e. the number of connections ' $c$ ' grows $\mathrm{O}\left(\mathrm{n}^{2}\right)$ with the number of nodes ' $\mathrm{n}$ ', 
making it impractical for large networks. The two architectures are shown below in Fig. 1.

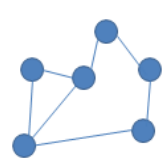

(a)

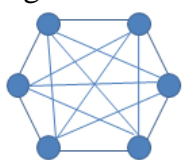

(b)
Figure 1. Partially connected vs fully connected mesh network.

In WSN, efficient routing protocols find routes for communication based on e.g., minimum energy, shortest path, number of hops etc. so every node need not to broadcast, thus saving energy and bandwidth simultaneously. The distributed nature of hierarchical network is effective for time critical applications. However the traditional routing protocols (e.g., AODV, OLSR, DSR, TORA etc.) for flat architecture need to be modified for hierarchical architecture [9]. Some routing protocols like ARC (Adaptive Routing using Clusters) exist for hierarchical architecture [8]. Routing protocols are out of scope of our present work.

It is important to note that for a single tier in distributed systems, each node have to communicate with all other nodes for local as well as global decisions. This means an added complexity and cost that will require long range communication, high bandwidth and a strict bound on delays. Therefore single tier architecture is not recommended for long range distributed applications. In [10], a hierarchical approach is used in a multi-level heterogeneous network connecting unmanned aerial vehicles (UAVs) which provide support for the multi-area theater with large number of ground units. There are 3 integrated information and communication $\left(\mathrm{I}^{2} \mathrm{C}\right)$ levels i.e. the ground radio interface for communications among ground nodes as well as between regular ground nodes to backbone nodes, the directional point-to-point wireless links among backbone nodes and a third radio interface for accessing UAV aerial backbone nodes. A 3-tier hierarchical architecture is proposed in [8] for improving the scalability properties of adhoc wireless networks. The proposed architecture includes sensor nodes (SN), Forwarding nodes (FN) and Access point (AP). The performance of traditional flat adhoc networks with a three-tier hierarchical approach as found to have improvements in performance and system capacity independent of routing protocols. However, for the hierarchical network, AODV perform better than DSR in terms of capacity and packet delay $[8,9]$. In addition, the modified routing metrics can be used to trade off throughput and delay against energy consumption. It was found that the three-tier hierarchical network's capacity scales as a function of the relative densities of forwarding nodes and access points [10]. These results indicate that it is possible to scale network capacity quite well with a mix of several radio forwarding nodes and just a few wired access points will be needed in the architecture.

In [11], a three-tiered architecture is presented for traffic information and monitoring based on $916 \mathrm{MHz}$ and
2.4 GHz radios in ISM band. The sensor network is used as first tier, transmission \& Management tier based on local leaders, while decision making tier as the highest one for deciding strategies based on information sent by lower tiers. Increasing number of tiers not only increases the complexity and cost but also takes more time for decision. In addition interference and security issues are common in such schemes. A decentralized two-tiered architecture in large-scale wireless sensor networks is described in [12], where an upper layer WLAN (802.11), serves as a backbone to an autonomous wireless sensor network based on 802.15.4. This approach is presented as a scalable, reliable, real-time and energy efficient scheme in the design of a two-tiered sensor network architecture without analyzing end-to-end delays in network communication. In addition, to extend long range communication, a number of WLAN nodes will be needed for multiple hops. In [13], a motes-master based 'Tenet' networking subsystem is considered. The multimode data fusion functionality and multi-node application logic should be implemented only in the master tier. The approach emphasizes on local processing of data instead of sending large sequences of time series data to upper layers. However, emphasis on local processing results in costly solution for scalable network.

\section{Multi Tier Generalised Architecture}

Consider a generalized architecture in which data passes through a number of channels/networks. The problem posed most often is to evaluate end-to-end performance in multiple networks. From acquisition to analysis, an embedded network is necessary for control and decisions based on multi sensor data. For distributed systems intelligence is not limited to one autonomous system which has full local knowledge but can contribute only a small part for global intelligence and decision making.

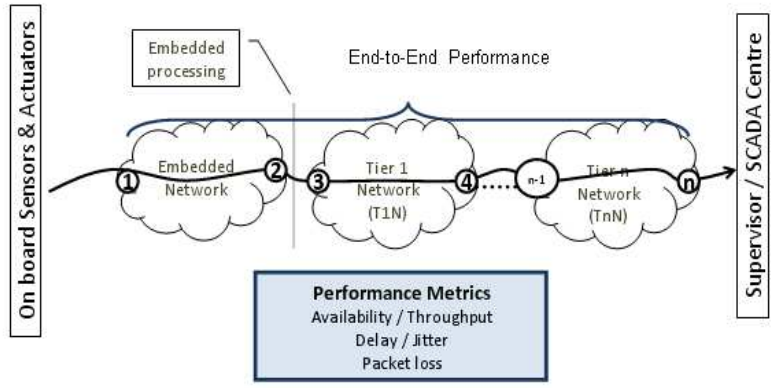

Figure 2. Multi tier heterogeneous network architecture.

As seen from the Fig. 2, every network adds some delay and bandwidth constraints in the hierarchy. So analysis in wireless network architecture is meant to analyze the complex end-to-end behavior. 


\section{A. 2 Tier Architecture}

The two-tier heterogeneous architecture is proposed here for a group of mobile robots on search and rescue $(\mathrm{S} \& \mathrm{R})$ mission. The low tier of the network consists of a set of mobile hosts which form a mesh network. Each mesh can communicate by the high tier through gateways, which can access to cellular/infrastructure networks. The bandwidth and reach of the outer tier should be suitable enough to allow long range control \& coordination applications. Providing high-speed data and telecommunications services, this architecture is evaluated for distributed applications with real time constraints on end-to-end delay and throughput [11]. Our goal is not to evaluate routing protocols for mobile ad hoc network (MANET) and their energy efficiency in the hierarchical architecture that is currently a popular area of research; instead, we are more oriented towards a centralized scenario for sensor network in which a leader node is receiving data from child nodes (acting as a sink node) and forwarding it to the external (backbone) network. In this way, every node can be accessed through the leader node, while leader nodes in each cluster exchange data of global interest in the network. This aspect is highlighted to simplify hardware needs for each mobile robot by assigning only one node to act as gateway (static in case of infrastructure based architecture) in each cluster. It is interesting to evaluate the resulting system capacity and the communication performance in case of time critical applications.

Our contribution is to use the existing infrastructure and protocols for the second tier that can guarantee end to end QoS, once the prioritized information is sent by the sensor network through the gateway for long range transfer.

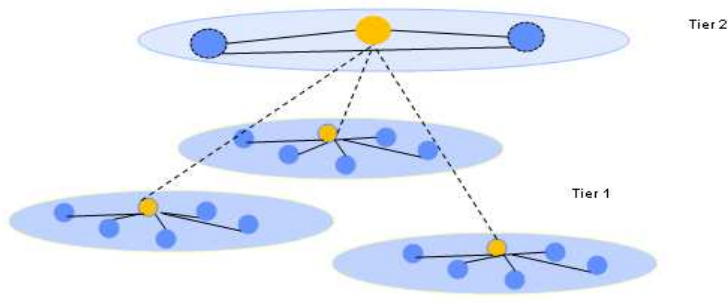

Figure 3. 2 tier network architecture.

Thus the network architecture is simplified as compared to the 3-tier hierarchical architecture without establishing a dedicated link as done in [11].

\section{B. Choice of protocol}

The choice of protocol in each tier is crucial w.r.t maintaining real time data transmission between the teams as well as among the team members.

\section{1) Tier 1 Protocol}

In many applications incorporating structural health monitoring, medical surveillance and habitat monitoring,
LR-WPANs (e.g., 802.15.4) are preferred due to their small size and low power consumption. However, if high data rate, security and bandwidth are required, WLAN (802.11) with some variations is the preferred choice for a mobile ad hoc as well as infrastructure based network. LRWPAN offers low data rate and at the same time require CFP (collision free period) management by the PAN coordinator for real time transmission [12].

2) Tier 2 Protocol

The second tier needs a long range communication protocol with extensive QoS support. Traditional mobile communication protocols e.g., GPRS offers QoS classes based on priority, reliability, delay and bandwidth required for the user applications. More rigid IP based QoS policies are proposed for GPRS/UMTS backbone network [13]. WiMAX is one of the strong candidates for tier 2 protocol with advantages being an IP-based network, relatively simple network topology than cellular networks, takes less equipment and less time to set up infrastructure with lower equipment and full mobility support for users [12]. WiMAX has both the options of frequency and time division duplexing (FDD and TDD); however, FDD does not provide the optimum use of resources. Whereas, with TDD, operators are capable of adjusting downlink and uplink ratios based on their service needs in the networks.

\section{QOS IN 2 TIER ARCHITECTURE}

Quality of Service (QoS) is usually considered as a combination of some key network characteristics i.e., Bandwidth, latency, jitter, packet loss and reliability that determines if a wireless technology can successfully deliver high value services (e.g., voice, data, video) [8]. On the other hand, there are applications which involve ensuring QoS at certain time interval for passing critical information; like, event based alarms in real time from one network node to another. In order to achieve this target we need to evaluate QoS parameters and mechanisms available in all subsequent networks involved in the communication path [14].

A simple case of 2 tier network is considered here. A sensor network utilizing 802.11 in the first tier while 802.16 (WiMAX) is considered in the second tier. The network architecture is analyzed to evaluate the suitability for real time data communication for control and collaboration over wireless network involving large distances. The generalized QoS consideration ensures that those packets sent through CFP on 802.11 should be allocated greater priority (e.g., rtPS) in WiMAX network to make real time message transmission possible [15].

\section{A. QoS provisions in 802.11:}

The basic WLAN is an important NGN access network but has least developed QoS mechanisms, due to its contention based medium access mechanisms [12]. In addition, the existing WLAN QoS schemes implement QoS on individual networks independently. Since IEEE 802.11 is connectionless, a host that sends a transmission cannot detect the state of the network or the state of the destination before transmission. The MAC layer defines 
two different access methods, the 'listen before transmission' or distributed coordination function (DCF) which is used for random access 'best-effort' traffic with several other algorithms specific to the WLAN. The point coordination function (PCF) uses contention free period (CFP). The IEEE 802.11 standard specifies that the basic technique DCF is mandatory and other procedures CSMA/CA with RTS/CTS and Hybrid coordination function (HCF) are optional [6]. HCF is more popular as it uses both by assigning different waiting periods to node with different real-time data transmission constraints. CFP is the $\mathrm{HCF}$ period and contention period $(\mathrm{CP})$ is the duration of contention in Fig. 4 below. Beacons or control frames are used to start superframes in CFP.

\begin{tabular}{|l|l|l|l|l|l|}
\multicolumn{1}{c}{$\boldsymbol{C F P}$} & \multicolumn{1}{c}{ CP } & \multicolumn{1}{c}{ CFP } & CP \\
\hline Beacon & PCF & DCF & Beacon & PCF & DCF \\
\hline
\end{tabular}

Figure 4. 802.11 Superframe format with CFP and CP periods [15].

Traffic on these network technologies, however, is subject to delay, making bandwidth availability and delivery time difficult to predict. Although high-priority traffic typically arrives at its destination before lowerpriority traffic as per demands of time-flexible telecom applications, it cannot be guaranteed to arrive within a specified time needed in time critical applications. Despite the fact that QoS is more difficult to implement on connectionless networks, there is growing interest in developing QoS for IP-based networks [16]. In connectionless networks, the two ends are not aware of the traffic being sent on the channel, for example User datagram protocol (UDP) in the transport layer which is easier to implement but not reliable as it maintain no state information and packet losses. On the other hand, Transmission control protocol (TCP) ensures reliable data transfer with error checking and reporting at both ends. Thus in Connection oriented networks e.g., WiMAX, establishing QoS is easier than connection less protocols. In 802.11e there are two co-ordination functions known as Hybrid Coordination Function (HCF). The first coordination function is the Enhanced Distributed Coordination function (EDCF), which is a QoS enabled version of DCF and the HCF Controlled Channel Access (HCCA), which is similar to the PCF [12]. In 802.11e, combined EDCF and HCF controlled channel access mechanisms are implemented for ensuring QoS in WLANs.

\section{B. QoS provisions in 802.16:}

IEEE 802.16 or WiMAX is an evolving set of wireless broadband standards focused on delivering high bandwidth wireless access service to subscribers over the "last mile". QoS is an essential brick of IEEE 802.16 as each connection is associated to a specific QoS. Management messages e.g., dynamic service addition (DSA) and dynamic service change (DSC) are used for maintaining QoS. In WiMAX, the latency on the wireless portion of a network is minimal relative to that on the wired portion of a network [15].
TABLE I. WIMAX SERVICE Classes

\begin{tabular}{|l|l|l|}
\hline Service Class & QoS Options & Typical Applications \\
\hline $\begin{array}{l}\text { Unsolicited } \\
\text { Grant Services } \\
\text { (UGS) }\end{array}$ & $\begin{array}{l}\text { RT fixed packet size, Jitter } \\
\text { \& Max latency tolerance, } \\
\text { Max sustained traffic rate }\end{array}$ & VoIP \\
\hline $\begin{array}{l}\text { Real time } \\
\text { packet service } \\
\text { (rtPS) }\end{array}$ & $\begin{array}{l}\text { RT variable packet size, } \\
\text { Traffic Priority and Max } \\
\text { latency tolerance }\end{array}$ & $\begin{array}{l}\text { Streaming } \\
\text { Audio/Video }\end{array}$ \\
\hline $\begin{array}{l}\text { Extended real } \\
\text { time packet } \\
\text { service (ErtPS) }\end{array}$ & $\begin{array}{l}\text { Traffic Priority, Jitter \& } \\
\text { Max latency tolerance }\end{array}$ & $\begin{array}{l}\text { VoIP (voice with } \\
\text { activity detection) }\end{array}$ \\
\hline $\begin{array}{l}\text { Non real time } \\
\text { packet service } \\
\text { (nrtPS) }\end{array}$ & $\begin{array}{l}\text { Traffic Priority, Min } \\
\text { reserved and Max } \\
\text { sustained traffic rate }\end{array}$ & FTP \\
\hline $\begin{array}{l}\text { Best Effort } \\
\text { (BE) }\end{array}$ & $\begin{array}{l}\text { Traffic Priority, Max } \\
\text { sustained rate, Traffic } \\
\text { Priority \& Request / } \\
\text { Transmission policy }\end{array}$ & $\begin{array}{l}\text { Data transfer, web } \\
\text { browsing }\end{array}$ \\
\hline
\end{tabular}

Five classes of service are supported as shown in the table 1 above with QoS options and typical applications.

\section{Hybrid QoS architecture}

In our network architecture, WLAN and WiMAX have different link-layer QoS mechanisms [12]. The goal of combining the QoS of these two networks involves looking at a hybrid architecture that is able to host both networks. This requires studying each network's link-layer QoS and then looking at the network control signaling required to make the networks work together. In [15] interactions between different layers and network entities are discussed to provide QoS when interworking the two protocols. A mapping between the two link-layer classes and the NGN DiffServ classes is usually required to operate at the network layer $[16,17]$.

The restriction on utilization in DiffServ networks is difficult to accommodate in some networks, therefore, many advanced architectures have been proposed for delay sensitive real-time applications. In fact, DiffServ over MPLS [16] cannot overcome the fundamental problem of DiffServ because the traffic engineering function is orthogonal to conventional DiffServ functions which realize a range of service classes by using different provisioning ratios per class [18, 19]. However, as indicated in [15], there is no analytical or experimental evidence to support the validity of this approach, so there is still room for further research in this area.

\section{APPLICATION SCENARIOS}

In remote monitoring, different events associated with the system under observation need to be sent for generating warning and corresponding necessary actions $[21,22]$. These applications include for example, observing seismic activity and volcano eruption in areas with a history of such incidents where volcanic eruptions are only observed approximately one hour before the magma reaches the surface [23]. With such a hard constraint, real-time transmission is necessary for early warning systems and preparation for evacuation. In addition, tsunami warning systems are also very crucial to detect tsunamis and issue warnings to prevent loss of life. 
It consists of two equally important tiers: a sensor network to detect tsunamis and a communications infrastructure to issue warning to local population.

Fire detection \& Prevention is another area of interest in such applications. One of the key success factors in this area of environmental monitoring is early fire detection, since an unattended fire ignition can result into uncontrollable proportions in short time. Heat sensing through motes and video surveillance with automatic detection of smoke or heat sources can augment the efficiency of the surveillance systems. The same network can also be used for coordination between the fire fighters and emergency work force to help limiting fire spread out to other areas. In power industry monitoring alarms need to be transferred in the least time possible to make or break a circuit, thus avoiding short circuits [24, 25].

\section{Simulation OF 2 TiER SCENARIO}

The scenario considered here is a team of mobile robots (as shown in Fig. 5 below), connected to WLAN network infrastructure. The 'sensor team' is equipped with sensors to take measurements, such as heat, vibration or radioactivity and update an application server over IP. These sensors may also include a camera to detect an object of interest and send images at regular intervals. Team 1 and 2 are rescue teams that will be put into action after analyzing sensor data or alarm. This type of architecture is useful in monitoring seismic and volcanic activity as well as for emergency services in case of natural hazards. For simplicity, the network is considered fixed infrastructure composed of WLAN/WiMAX gateway nodes with WiMAX as tier 2 network backbone. For simplicity, node mobility is not considered.

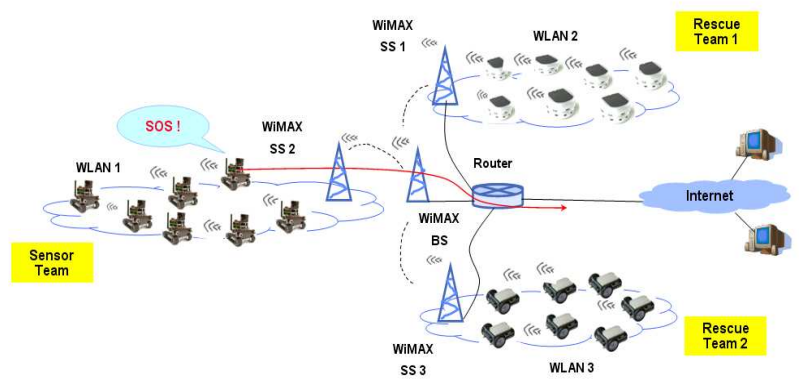

Figure 5. Heterogeneous network architecture for distributed coordination and control of mobile robots.

The WLAN/WiMAX gateways are communicating with the WiMAX base stations which are further connected to an application server via Ethernet and simulated in OPNET [26]. Two profiles are defined for the nodes in simulation i.e. normal application profile and the 'SOS' profile. Each node in the subnet is configured for a normal user profile which sends a heavy FTP and HTTP application to the application server. Each FTP application has a size of $500 \mathrm{~KB}$. The time between each node's file transfer requests, is exponentially distributed with mean as 360 seconds. The page inter-arrival times are exponentially distributed with a mean of 60 seconds. Each page has 1000 bytes of text with 5 'medium images' each with size randomly picked with a uniform distribution on [500, 2000] bytes. HTTP application size in our scenario is taken as 1000 bytes with 10 medium images. However, for node 4 in the sensor team, a light FTP application with highest priority is defined with the usual heavy http flow on the WiMAX. This prioritized light FTP traffic actually simulates 'SOS' or urgent messages with a file size of 1000 bytes.

The results are shown in Fig. $6 \& 7$ (at the end) below which represents end-to-end delay for FTP application and the throughput in sensor subnet respectively. It can be observed that node- 4 prioritized rtPS flow starts at $8 \mathrm{sec}$ and it has the minimum end-to-end average delay of about $9 \mathrm{~ms}$ for SOS message.

\section{CONCLUSION}

This paper presents heterogeneous network architecture for distributed control and coordination purposes. Delay characteristics for critical data transfer in WLAN networks connected through gateway nodes to a broadband network is evaluated. As a next step, synchronized QoS service management combining the 2 tiers and incorporating IP QoS options will be purposed to guarantee end-to-end delivery and minimum latency despite heavy loads over network.

\section{ACKNOWLEDGMENT}

This work is supported by HEC (Pakistan) under OSS06 program. We are also thankful to OPNET Technologies, Inc. for their active support under OPNET University Program (OUP).

\section{REFERENCES}

[1] M.Y. Chow and Y. Tipsuwan, "Gain Adaptation of Networked DC Motor Controllers on QoS Variations", IEEE Transactions on Industrial Electronics, Vol. 50, no. 5, October, 2003.

[2] C.E. Houstics, "Module allocation of real-time applications to distributed systems", IEEE Transactions on Software Engineering,, Jul 1990, vol. 16, Issue: 7, pp. 699-709.

[3] Amine Mechraoui, Zeashan H Khan, Jean-Marc Thiriet and Sylviane Gentil, "Co-design for wireless networked control of an intelligent mobile robot", 6th international conference on informatics in control, automation \& Robotics, ICINCO 09, Italy.

[4] H. Chan and Ü. Özgüner, "Closed-loop control of systems over a communications network with queues", Int. J. Control, vol. 62, no. 3, pp. 493-510, 1995.

[5] ITU-T Recommendation Y.2012, Functional Requirements and Architecture of the NGN, 2006. http://www.itu.int/ITU-T/

[6] Mario Marchese, QoS Over Heterogeneous Networks, Wiley, 07.

[7] ITU-T Recommendation Y.1541, Network Performance Objectives for IP-Based Services, Feb. 2006. http://www.itu.int/ITU-T/

[8] S. Zhao, K. Tepe, I. Seskar, and D. Raychaudhuri, "Routing Protocols for Self-Organizing Hierarchical Ad-Hoc Wireless Networks", Proc. IEEE Sarnoff 2003 Symposium, March 2003.

[9] E.M. Belding-Royer, "Hierarchical Routing in Ad hoc Mobile Networks", Wireless Communication and Mobile Computing, pp. 515-532, 2002.

[10] D.L Gu,. Pei Ly Guangyu, H. Gerla and M. Xiaoyan Hong, "Hierarchical routing for multi-layer ad-hoc wireless networks 
with UAVs", Proc. of 21st Century Military Communications Conference, MILCOM 2000, Los Angeles, vol.1, October 22-25, 2002, pp. 310-314.

[11] Mingchen Zhang, Jingyan Song and Yi Zhang, "Three-tiered sensor networks architecture for traffic information monitoring and processing", IEEE/RSJ International Conference on Intelligent Robots and Systems, IROS 05. 2-6 Aug. 2005 pp. 2291 - 2296.

[12] Anis KOUBAA and Mario Alves, "A Two-Tiered Architecture for Real-Time Communications in Large-Scale Wireless Sensor Networks: Research Challenges”, ECRTS'05, Spain, 2005.

[13] O. Gnawali, B. Greenstein, Ki-Young Jang, August Joki, J. Paek, M. Vieira, D. Estrin, R. Govindan and E. Kohler, "The Tenet Architecture for Tiered Sensor Networks", Proc. of the 4th international conference on Embedded networked sensor systems, Boulder, Colorado, USA, pp: 153 - 166, 2006.

[14] D. Clark, S. Shenker, and L. Zhang, "Supporting Real-Time Applications in an Integrated Services Packet Network: Architecture and Mechanism”, Proc. SIGCOMM, Sept. 1992, pp. 14-26.

[15] H. Labiod, H. Afifi and C. De Santis, Wi-Fi, Bluetooth, Zigbee and WiMAX, Springer, 2007.

[16] F. Agharebparast and V.C.M Leung, "QoS support in the UMTS/GPRS backbone network using DiffServ", Global Telecommunications Conference, 2002. GLOBECOM 02. IEEE 17-21 Nov. 2002, Vol. 2, pp: 1440- 1444.

[17] Y. Jia and M. Chen, "A New Architecture of Providing End-to-End Quality-of-Service for Differentiated Services Network", IEEE Military Communications Conference-MILCOM 2001, October 2001, pp. 1451-1456.

[18] Humaira Haffajee and H. Anthony Chan, "Low-cost QoS-enabled Wireless Network with Interworked WLAN and WiMAX", proceedings of the First IEEE International Conference on Wireless Broadband and Ultra Wideband Communications (AusWireless 2006), Sydney, March 13-16, 2006.

[19] J. Joung, "Feasibility of Supporting Real-Time Traffic in DiffServ Architecture", Proc. of 5th Int'l Conf. on Wireless/Wired Internet Communications (WWIC), vol. 4517, May 2007, pp. 189-200.

[20] S. Oueslati and J. Roberts, "A New Direction for Quality of Service: Flow-Aware Networking", Proc. Conference on Next Generation Internet Networks (NGI), April 2005, pp. 226-232.

[21] F. Xia, Y. C. Tian, Y. Li and Y. Sun, "Wireless Sensor/Actuator Network Design for Mobile Control Applications", Sensors 2007, 7, 2157-2173.

[22] H. J. Koerber, H. Wattar and G. Scholl, "Modular Wireless RealTime Sensor/Actuator Network for Factory Automation Applications", IEEE Trans. on Industrial Informatics 2007, 3(2), 111-119.

[23] Marcoz Katz and F. Fitzek, WiMAX Evolution: Emerging technologies \& Applications, John Wiley \& Sons, 09.

[24] Zeashan H.Khan, Denis G. Catalot and J.M. Thiriet, "Wireless Network architecture for Diagnosis and Monitoring Applications", CCNC 2009, Jan 11-13, 09, Las Vegas, USA.

[25] M. Haffar, Z. H. Khan, Jean-Marc Thiriet and Eric Savary, "An extension to IEC 61850 for solving selectivity problem in electrical substations", IAR/ACD, UK, Nov 08.

[26] OPNET Technologies, Inc. http://www.opnet.com/ [May 25, 2009].

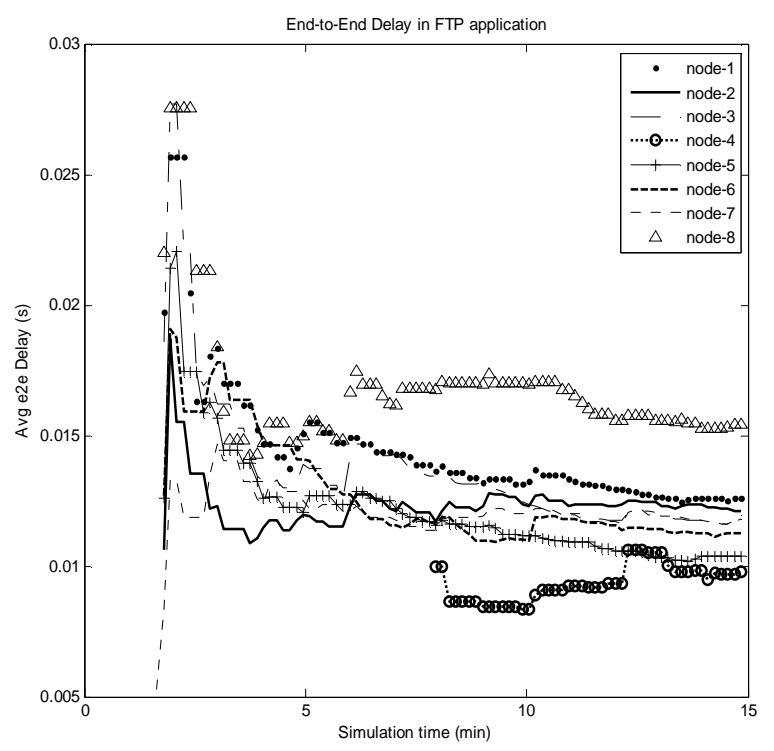

Figure 6. End-to-End delay in FTP application (sensor team).

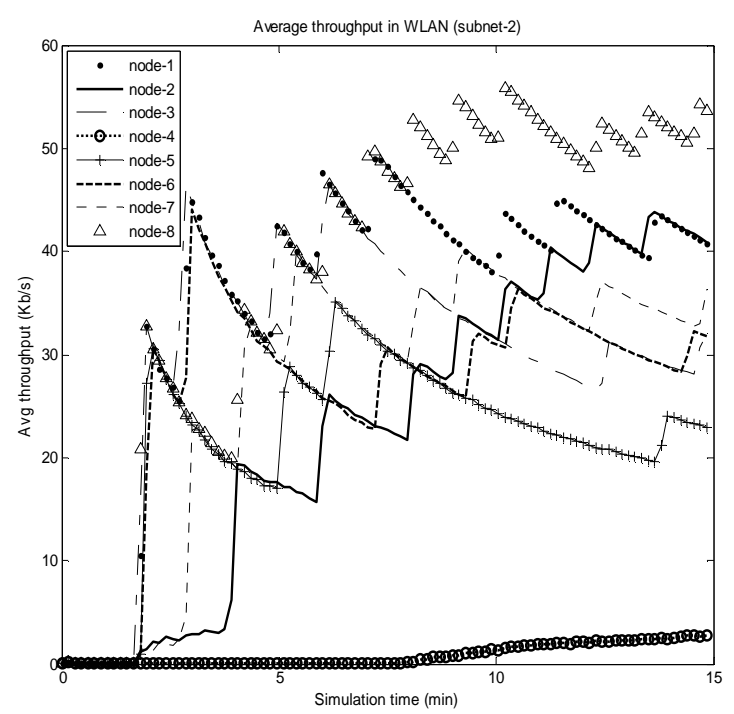

Figure 7. Average throughput in WLAN. 